\title{
Understanding the Awareness and Practice of Hand Washing among Diverse Population
}

\author{
R. R. Snehalakshmi ${ }^{1}$, A. R. Susethira ${ }^{2^{*}}$, N. Prabhusaran $^{1}$ and A. Uma ${ }^{1}$ \\ ${ }^{1}$ Department of Microbiology, Trichy SRM Medical College Hospital and Research Centre, \\ Tiruchirapalli, India (Affiliated to The Tamilnadu Dr. M.G.R. Medical University, Chennai) \\ ${ }^{2}$ Department of Microbiology, Government IRT Perundurai Medical College, Erode, India \\ (Affiliated to The Tamilnadu Dr. M.G.R. Medical University, Chennai) \\ *Corresponding author
}

\section{Keywords}

Hand washing, KAP, Heterogenous population

Article Info

Accepted:

15 December 2019

Available Online:

20 January 2020

\section{A B S T R A C T}

Hand washing prevents the transmission of infections including waterborne, parasitic and nosocomial infections. Hand washing is the most effective and cheaper method of preventing the spread of germs. The main aim of the study is to evaluate the knowledge, attitude, behavior and practice of hand washing among the heterogeneous population including Doctors, Nurses, Medical students, Paramedical staffs, Non-Medical students, School children and mothers of under-five in Tiruchirappalli, Tamilnadu. Method of study is cross-sectional study, study conducted for 2 months from June-August 2015. Among the 400 participants, 10\% doctors, $7.5 \%$ nurses, $25 \%$ medical students, $7.5 \%$ Para-medical staffs, $25 \%$ Nonmedical students, $12.5 \%$ school children and $12.5 \%$ mothers of under-five were participated. The data were analyzed and found that the knowledge among doctors, nurses, medical students, Para-medical staffs, Non-medical students, school children and mothers was $64.5 \%, 58.8 \%, 50.7 \%, 57.6 \%, 38.28 \%, 49.3 \%$ and $39.5 \%$ respectively. The attitude among doctors, nurses, medical students, Para-medical staffs, Non-medical students, school children and mothers was $95.7 \%, 72.8 \%, 89.1 \%, 52.2 \%, 83 \%, 82.4 \%$ and $63.1 \%$ respectively. The practice of hand washing among, nurses, medical students, Para-medical staffs, Nonmedical students, school children and mothers was $63.1 \%, 51.6 \%, 51.6 \%, 51.2 \%$, $44.2 \%, 44 \%$ and $45 \%$ respectively. For medical professionals it is mandatory to wash hands before and after touching patients and after handling infectious materials but this survey didn't reveal the expected results from the healthcare professionals and others. Hence the hand washing procedures should be included in the curriculum from the elementary level to higher level. 


\section{Introduction}

The World health organization has declared October $15^{\text {th }}$ as a Global hand washing day to emphasize the importance of hand washing to all and particularly by the health care professionals (WHO, 2014). On hand hygiene day, WHO insist health care providers to practice good hand hygiene and taking care of patients. The WHO educates 5 moments for hand hygiene which are before touching a patient; before clean aseptic procedures; after contact with body fluids; after touching the patients and after touching patient's surroundings (WHO-Good hand hygiene by healthcare workers protect patients from drug resistant infection 2014). Hands frequently become contaminated which respiratory viruses either directly or through contact with contaminated surfaces (Ansari et al., 2014). Most of the infections have been scientifically proved that it is transmitted through improper hand hygiene.

Globally, developing countries have been burdened with infectious diseases and millions of deaths reported annually due to poor sanitation and unhygienic practices. Among the infectious moiety, acute respiratory disorders and diarrhea play an important role in increasing the mortality. Many studies and meta-analysis have shown that simple method of Hand washing with soap (HWWS) which act as a barrier in transmitting the infection and cost effective (Seema et al., 2014; Datta et al., 2011). Also the practice of HWWS could curtail down both diarrheal and respiratory infections by $47 \%$ and $23 \%$ respectively (Luby et al., 2005; Rabie and Curtis, 2006). Good hand washing practices is rare in community setting and awareness is meager among low and middle socio economic status (Datta et al., 2011). In India hand washing practice has not been noticed as an essential means by health care authorities when compared to Oral
Rehydration Therapy (ORT), Anti-Retroviral therapy (ART) and standard case management in diarrhea.

The proper hand washing would be a significant impeded technique reducing various incidences of infectious diseases. Cleaning hands by the health care workers reduced mortality in various situations. Lack of resources, namely soap and water, as well as inadequate sanitation facilities are the main reasons where all do not wash their hands. In addition to having proper Resources and facilities, hygiene practices are heavily influenced by knowledge and attitudes towards hygiene (Vivas et al., 2010). It is not possible by the young children to wash their hands on own, this can't prevent the transfer of germs between their hands and mouth. Adequate hand washing after fecal contact is failed by most of the mothers in both developed and developing countries (Datta $e t$ al., 2011).

Hand washing practice along with plain or antimicrobial soap and water by the mothers can curtail the childhood morbidity and washing hands only with water is not sufficient (Datta et al., 2011, Seema et al., 2014; Opara et al., 2009). Consultants washing their hands between patients can reduce the nosocomial infections. Its prevalence is about $5 \%$ \& $25 \%$ in developed and developing countries respectively (Opara et al., 2009; Harsha et al., 2013; Ali et al., 2014) Patients in ICU setup has more chance of being infected with multi-drug resistant organism and the mode of spread is mainly by the contaminated hands of consultants (Shiddharth et al., 2015). Nosocomial infections mainly occur by the contaminated hands of healthcare workers (Khaled et al., 2008).

Due to poor hand washing incidence of Gastro-enteric infections, respiratory 
infections, eye infections, skin infections have been increased (Opara et al., 2009; Loria et al., 2005; Curtis et al., 2003). Hence in order to study the hand washing knowledge, practice, attitude and behavior among the medical and Para-medical professionals, axillary healthcare workers, medical students, engineering students, school students, mothers of under five children were studied through a questionnaire survey to assess the knowledge, practice, attitude and behavior of hand washing and indirectly educate them the importance of hand washing. The present study is done to ascertain the knowledge and practice of hand washing in heterogeneous population in Tiruchirapalli, Tamilnadu. A secondary objective is formulated to identify the factors associated with hand hygiene.

\section{Materials and Methods}

This is a cross sectional, prospective and questionnaire study conducted for the period of 2 months at tertiary care hospital at Tiruchirapalli, India. A total of 450 individuals which includes 50 mothers; 100 each in school children, college students (medical and non medical) and health care workers. As the study is non invasive and KAP survey; thus verbal consent will be obtained from the participants.

The following procedures and activities will be carried out. The workers will be subjected to an interviewer guided questionnaire which probed into demographic data and information on knowledge, attitude and practice. It is designed to be completed within 15 minutes for an average respondent. The language used is vernacular language in which the mothers tongue of the individuals. Any technical terms are translated and explained by the interviewer. The interview will be conducted by one of the researchers throughout to avoid the problem of interinterviewer variations.

\section{Results and Discussion}

The study was conducted among 500 persons of heterogeneous population comprising of Doctors, Nurses, Medical students, Paramedical staffs, non-medical students, school children and mothers. Of them only 400 were responded to the survey and the response rate is $80 \%$. The questionnaire of the responded persons was analyzed from the study. Out of 400 participants, Doctors comprised of $10 \%$, Nurses $7.5 \%$, Medical students 25\%, Non-Medical students $25 \%$, School children $12.5 \%$, Mothers $12.5 \%$ and Paramedical staffs $7.5 \%$ (Figure 1).

When the data is analyzed it shows that $64.5 \%$ of doctors, $58.8 \%$ nurses, $50.7 \%$ of medical students, $49.3 \%$ of school children and $39.5 \%$ mothers were having knowledge on hand washing. $61.72 \%$ of non-medical students and $60.5 \%$ of mothers were not having adequate knowledge of hand washing (Figure 2). Regarding attitude on hand washing $95.7 \%$ of doctors, $89.1 \%$ of medical students, $83 \%$ of non-medical students, $82.4 \%$ of school students were having attitude above $80 \%$. Nurses, paramedical staffs, mothers were having $72.8 \%, 52.2 \%$ and $63.1 \%$ respectively (Figure 3 ).

When the behavior of heterogeneous population were analyzed it was found that $54 \%$ of mothers used to scold the children for not washing their hands and interestingly $6 \%$ of medical students also used to scold for not hand washing and more interestingly $2.5 \%$ of doctors used to scold the children for not hand washing. Among the mothers $12 \%$ used to beat the children for not hand washing, $8 \%$ of non-medical students and $2 \%$ of the medical students used to beat the children of their home for not washing their hands. $100 \%$ of nurses and paramedical staffs used to teach the consequences of not hand washing to others as this is the professional ethics to 
teach the patients attending them. When compared with nurses only $97.5 \%$ of doctors used to teach the consequences of not hand washing, more interestingly $96 \%$ of the school students used to teach other students the consequences of not hand washing.

The teaching habit of mothers is very poor and only $34 \%$ as most of them prefer to scold and beat their child for not hand washing. $4 \%$ of school students were having the behavior of scolding and teaching the consequences of not washing hands. All the participants were having the behavior of teaching the benefits of hand washing to others which ranges from $92 \%$ to $100 \%$. When the soap is not available in the toilet the behavior varies, $15 \%$ of doctors, $20 \%$ of medical students, $15 \%$ of non-medical students, $22 \%$ of school children, $13.3 \%$ nurses and $6.7 \%$ of paramedical staffs were having the behavior of informing the authority to place soap in the toilet, interestingly $100 \%$ of mothers and $93.3 \%$ of paramedical staffs came forward to arrange soap for themselves in the toilet. $85 \%$ of the doctors, $86.7 \%$ nurses, $80 \%$ of medical students, $85 \%$ of the non-medical students and $74 \%$ of school students were having the behavior to arrange soaps for themselves in the toilet.

When the behavior of hand washing after playing was questioned $100 \%$ of nurses, $100 \%$ of paramedical staffs and $100 \%$ of mothers came forward to educate the benefits of hand washing and $95 \%$ of doctors, $97 \%$ of non-medical students, $95 \%$ of medical students and $92 \%$ of school children were also having the behavior of educating the benefits of hand washing to others.

When the practice of hand washing is analyzed among heterogeneous population it is found that $63.1 \%$ doctors, $51.6 \%$ of nurses, $51.6 \%$ of medical students, $51.2 \%$ of Paramedical workers had proper hand washing practice, whereas the practice of hand washing among Non-medical students, School children and mothers is about $44.2 \%, 44 \%$ and $45 \%$ respectively (Figure 4 ).

Table.1

Figure.1 Heterogeneous population included in the study

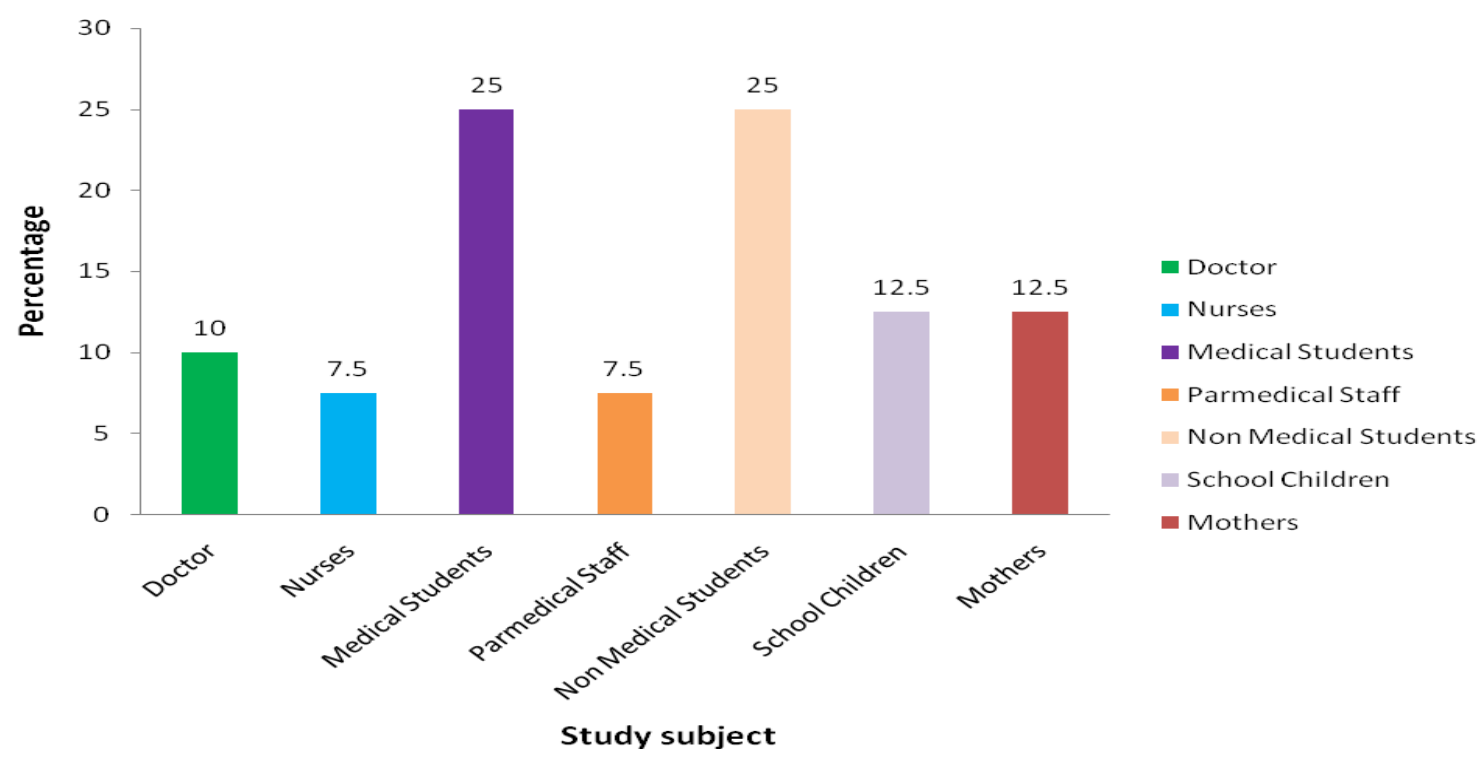


Figure.2 Information related to knowledge on hand washing

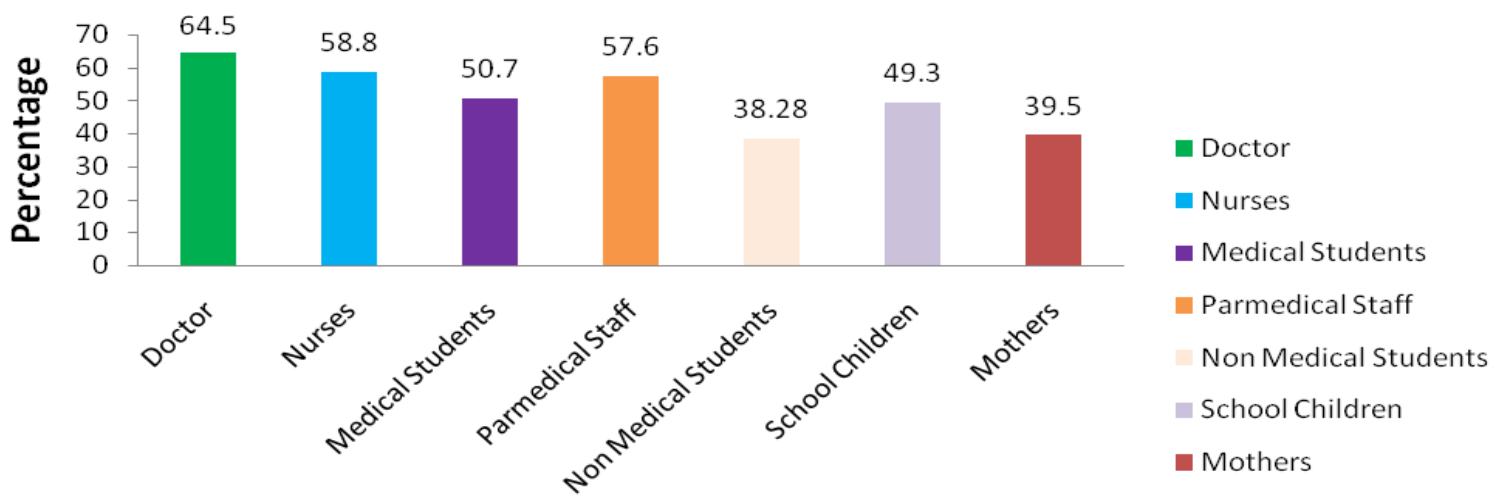

\section{Category of Participants}

Figure.3 Attitude on hand washing

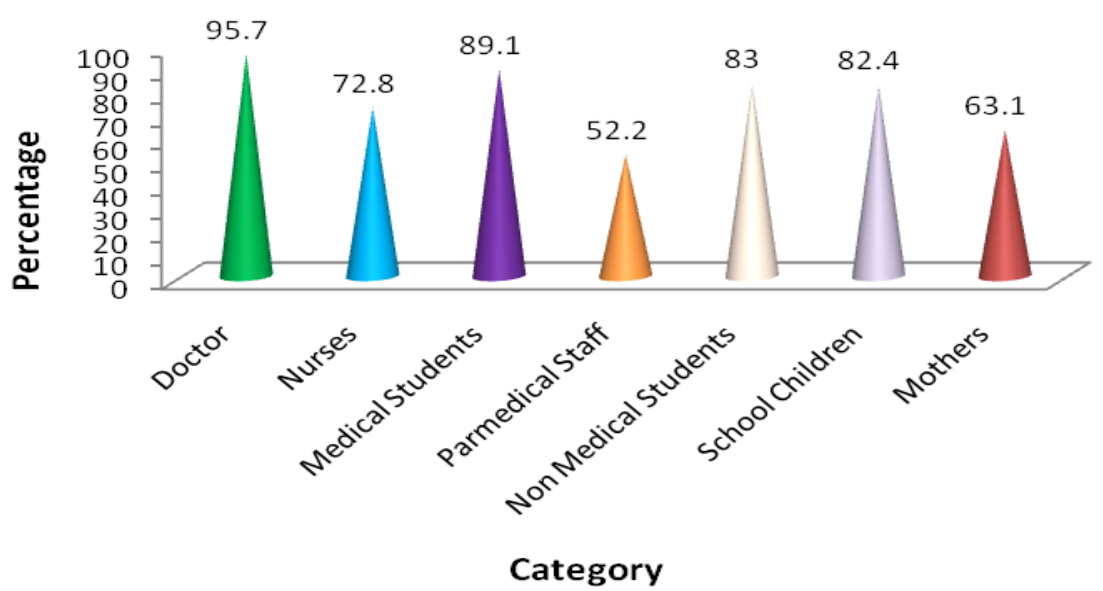

$$
\begin{aligned}
& \text { Doctor } \\
& \text { Nurses } \\
& \text { Medical Students } \\
& \text { Parmedical Staff } \\
& \text { Non Medical Students } \\
& \text { School Children } \\
& \text { Mothers }
\end{aligned}
$$

Figure.4 Practice of Hand washing

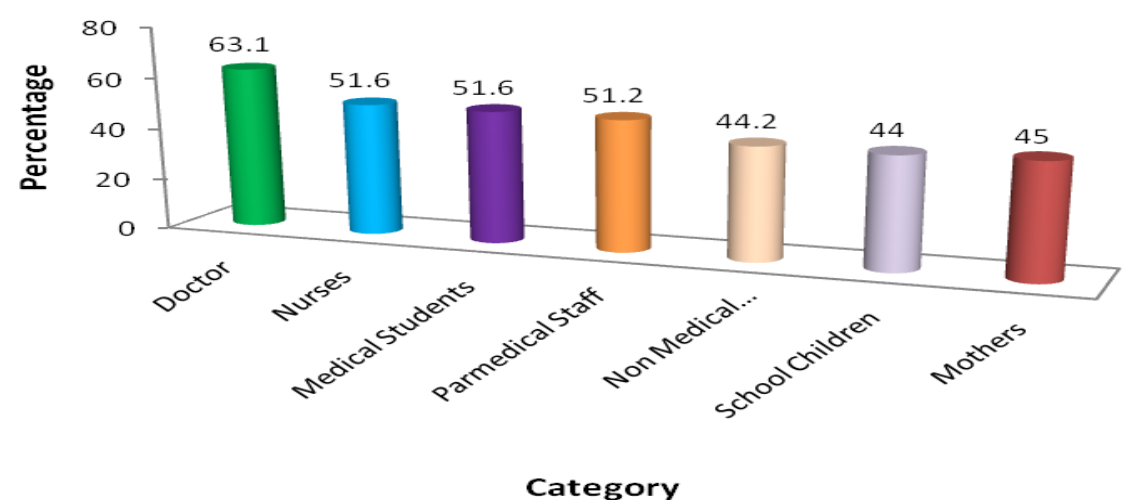

$$
\begin{aligned}
& \text { Doctor } \\
& \text { Nurses } \\
& \text { - Medical Students } \\
& \text { Parmedical Staff } \\
& \text { Non Medical Students } \\
& \text { School Children } \\
& \text { - Mothers }
\end{aligned}
$$


Hand washing is the practice of maintaining personal hygiene. The practice of hand washing should be thought from childhood itself by the parents and should be emphasized by the teachers to the students. Hand washing practice will prevent the spread of waterborne, Parasitic and Nosocomial infections. Hand washing is defined as washing hands with unmedicated detergent and water or water alone (Didier et al., 2001).

In a study conducted in a hospital in Cairo were found that $37.5 \%$ of doctors had practice of hand washing (Khaled et al., 2008). Another study at Mangalore, India revealed that $98.8 \%$ of doctors were having knowledge on hand washing (Harsha et al., 2013). A study among doctors about the knowledge is found to be $43.9 \%$ and practice is about $48.2 \%$ (Vivas et al., 2010). In our study it was found that knowledge, attitude and practice of hand washing among doctors is about $64.5 \%$, $95.7 \%$ and $63.1 \%$ respectively.

In a study, $11 \%$ of nurses were having good knowledge of hand washing, $10 \%$ of nurses were having good attitude on hand washing and $14 \%$ of nurses were having good practice of hand washing (Harsha et al., 2013). Study in which knowledge of hand washing among nurses was found to be $93.1 \%$ (Harsha et al., 2013). Studies said that $36.4 \%$ of nurses have good practice of hand washing (Khaled et al., 2008). Whereas studies regarding hand washing compliance among nurses is about $74.2 \%$ (Muhammad et al., 2012). Studies conducted about the knowledge of hand washing are about $91 \%$ and practice is about $20 \%$ in nurses (Phukar et al., 2014). In our studies it was found that knowledge, attitude and practice of hand washing among nurses is about $58.8 \%, 72.8 \%$ and $51.6 \%$ respectively.

A study stated that $8 \%$ of medical students were having good knowledge of hand washing, $1.5 \%$ of medical students were having good attitude on hand washing and $2 \%$ of medical students were having good practice of hand washing. Studies about hand washing practices among medical students in Nigeria, it revealed that $61.3 \%$ of medical students used to practice hand washing after day's work, $37.6 \%$ washed their hands regularly after interacting with patients (Opara et al., 2009). Studies at Saudi Arabia noted that $56 \%$ of the medical students having positive indications for hand hygiene and in another studies conducted at Royal London Hospital and School of Medicine and Dentistry in UK found that $8.5 \%$ of candidates washed their hands after patient contact (Khaled et al., 2008). In our studies it was found that $50.7 \%$ of medical students had knowledge on hand washing, $89.1 \%$ of medical students had attitude on hand washing and $51.6 \%$ of medical students has practice of hand washing.

Studies related to knowledge of hand washing are about $83 \%$ among Para-medical staffs and none of the paramedical staffs has practice of hand washing (Phukan et al., 2014). In our studies the knowledge, attitude and practice of hand washing is about $57.6 \%, 52.2 \%$ and51.2\% respectively. Studies said that practice of hand washing among Non-Medical students is about $54 \%$ whereas in our study the knowledge, attitude and practice of hand washing is about $38.28 \%, 83 \%$ and $44.2 \%$ respectively (Taylor et al., 2010). Studies on college students about knowledge, attitude, behavior and practice of hand washing were found to be very few and future studies are required to assess the KABP among college students.

A study among school children found that $33.6 \%$ has proper hand washing practice and the level of knowledge among medical students is about $52.1 \%$ (Curtis et al., 2003). Studies said that the practice of hand washing with soap is about $32 \%$ among grade 1 
students and about $80 \%$ in grade 7 students (Muhammad et al., 2012). In our studies the knowledge, attitude and practice of hand washing among school children is about $49.3 \%, 82.4 \%$ and $44 \%$ respectively.

Another study observed that $73 \%$ of mothers didn't wash their hands after cleaning child's defecation, $22 \%$ wash their hands with water and $2 \%$ with soap and water (Azzam et al., 2012; Le et al., 2013; Beth et al., 2007). A study observed that $83.41 \%$ of mothers were having knowledge on hand washing an aware that hand washing could prevent the spread of infections. But in our study it was observed that $39.5 \%$ of mothers were having knowledge on hand washing, $63.1 \%$ having attitude on hand washing, $45 \%$ having practice of hand washing.

In conclusion, the study revealed that the knowledge, attitude, behavior and practice of hand washing among Doctors, Nurses, Medical students, Para-Medical workers, Non-Medical students, School Children and Mothers were not up to the expected level. Hence continuous medical education (CME) on hand washing should be conducted for Medicals and Para-Medicals and importance of hand washing should be included in the curriculum. Hand washing for all occasions is felt essential to keep away the germs from contaminating oneself and others. For medical professionals it is mandatory to wash hands before and after touching patients and after handling infectious materials but this survey didn't reveal the expected results from the healthcare professionals and others. Hence the hand washing procedures should be included in the curriculum from the elementary level to higher level.

\section{Acknowledgment}

The Authors acknowledge all the participants who are supported and also thanked the
Indian Council of Medical Research (ICMR) for financial support under the scheme of short term studentships (STS).

\section{References}

Ali, M., and Mohamad, H.S. 2014. Hand washing study in health care workers of tertiary teaching hospital in Iran. Eur. J. Prev. Med. 2, 16-19.

Ansari, S.A., Springthorpe, V.S., Sattar, S.A., Rivard S., and Rahman, M. 2014. Potential role of hands in the spread of respiratory viral infections: studies with human parainfluenza virus 3 and rhinovirus. J. Clin. Microbiol. 29, 21152119.

Azzam, A.K., and Sajad, A.S. 2012. Hand hygiene practice among Medical students. Interdisc. Persp. Infect. Dis. 2012, 679-683.

Beth, E.S., David, W.L, and Val, C. 2007. Hard to handle: understanding mothers handwashing behavior in Ghana. Hlth Policy Planning. 22, 216-224

Curtis, V., and Cairncross, S. 2003. Effect of washing hands with soap on diarrhoea risk in the community: a systematic review. Lancet Infect. Dis. 3, 275-281.

Datta, S.S., Boratne, A.V., Singh, Z., Senthilvel, V., Natesan, M., and Gajera, $\mathrm{V}$. 2011. Influence of mothers' handwashing practices and availability of water and sanitary latrine on under five morbidity. Ind. J. Med. Spec. 2, 330-337.

Didier, P. 2001. Improving Adherence to hand hygiene practice: A Multidisciplinary approach. CDC Emerg. Infect. Dis. J. 7, 234-240.

Harsha, K.N., and Devi, D. 2013. An epidemiological study on hand washing practices among health care workers in hospitals of Mangalore city. Nat. J. Comm. Med. 4, 213-218.

Khaled, M.A.E., and Iman, M.B. 2008. 
Assessment of Knowledge, attitude and practice of hand washing among health care workers in Ain Shams University hospitals in Cairo - cross sectional study. Egypt. J. Comm. Med. 26, 19-25.

Kyle, T.J., Roselyne, B., Aya, Z., Chelsea, W. 2010. Hand Hygiene knowledge of college students. J. Am. Soc. Med. Technol. 23, 89-93.

Le, T.T.X., Luu, N.H. 2013. Hand washing among school children in an ethnically diverse population in northern rural Vietnam. Glob. Hlth. Action. 6, 1.

Loria, F., Kaufmann, R.B., Kay, D., Enanoria, W., Haller, L., Dan Colford, J.M.C., and Walter S. 2005. Sanitation and hygiene interventions to reduce diarrhea in less developed countries: a systemic review and meta analysis. The Lancet. 5, 42-52.

Luby, S., Agboatwalla, M., Feikin, D., Painter, J., Billhimer, W., and Altaf, A. 2005. Effect of handwashing on child health: a randomized controlled trial. The Lancet. 366, 225-233.

Muhammad, W., Darawad, R.N., Mahmoud, A.H., Iyad, I.A.N, Manal, A.S. (2012). Investigating Jordanian nurses handwashing beliefs, attitude and compliance. Amer. J. Infect. Contr. 17, 176-183.

Opara, P.I., and Alex, P.A.H. 2009. Hand washing practices amongst medical students in Port Harcourt, Nigeria. Nig. Hlth. J. 9, 14-24.

Phukan, P. 2014. Compliance to occasional safety measures among the Para-medical workers in a Tertiary Hospital in Karnataka, South India. Int. J. Occup. Environ. Med. 5, 40-50.

Rabie, T., and Curtis, V. 2006. Evidence that handwashing prevents respiratory tract infection: a systematic review. Trop. Med. Int. Hlth. 11, 1-10.

Seema, A.K., Miti, J.O., Vidya, P., Prafulla, S., and Uday, N.Y. 2014. Hand washing knowledge and practice among mothers of under five children in coastal Karnataka, India - a cross sectional study. Int. J. Med. Hlth. Sci. 3, 266-271.

Shiddharth, C., Varun, M., and Urvi, S. 2014. Hand hygiene compliance among healthcare workers in a accredited tertiary care hospital. Indian J. Crit. Care Med. 18, 223-229.

Vivas, A., Gelaye, B., Aboset, N., Kunie, A., Berhane, Y., and Williams, M.A. 2010. Knowledge, attitudes and practices (KAP) of hygiene among school children in Angolela, Ethiopia. J. Prev. Med. Hyg. 51, 73-79.

WHO. 2014. Good hand hygiene by healthcare workers protect patients from drug resistant infection.

\section{How to cite this article:}

Snehalakshmi, R. R., A. R. Susethira, N. Prabhusaran and Uma, A. 2020. Understanding the Awareness and Practice of Hand Washing among Diverse Population. Int.J.Curr.Microbiol.App.Sci. 9(01): 1935-1942. doi: https://doi.org/10.20546/ijcmas.2020.901.217 